\title{
Diffractive Physics at the Tevatron
}

\author{
K. Hatakeyama \\ For the CDF and D $\varnothing$ collaborations
}

The Rockefeller University, 1230 York Avenue, New York, NY, 10021, U.S.A.

\begin{abstract}
Experimental results of hard single diffraction and double pomeron exchange studies at the Fermilab Tevatron $p \bar{p}$ collider are presented. Single diffraction results are compared with predictions from phenomenological models and expectations from results obtained in diffractive deep inelastic scattering experiments at the DESY $e p$ collider HERA. Double pomeron exchange results are compared with corresponding single diffraction results to test factorization.
\end{abstract}

\section{INTRODUCTION}

Recent results from studies of hard single diffraction (SD) $[1,2]$ and double pomeron exchange (DPE) [3] events produced in $p \bar{p}$ collisions at the Fermilab Tevatron collider by the CDF and D $\varnothing$ collaborations are presented. Hard SD events contain a hard scattering and a leading (anti)proton associated with a rapidity gap, which is defined as a pseudorapidity [4] region devoid of particles. In hard DPE events, both incoming proton and antiproton are scattered quasielastically and are separated by rapidity gaps from a hard scattering occurring in the central region. The rapidity gap is presumed to be due to the exchange of a Pomeron $(\mathbb{P})$, which is a color-singlet state with vacuum quantum numbers. In this framework, a $p \bar{p}$ hard SD event can be expressed as $\bar{p}+p \rightarrow\left[\bar{p}^{\prime}+\mathbb{P}\right]+p \rightarrow \bar{p}^{\prime}+(j j, W, b \bar{b}, \ldots)+X$, and similarly, a $p \bar{p}$ hard DPE event as $\bar{p}+p \rightarrow\left[\bar{p}^{\prime}+\mathbb{P}\right]+\left[p^{\prime}+\mathbb{P}\right] \rightarrow \bar{p}^{\prime}+p^{\prime}+(j j, W, b \bar{b}, \ldots)+X$. These processes are illustrated in figure 1 .
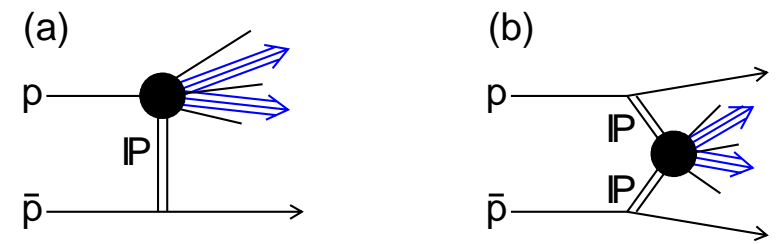

FIGURE 1. Diagrams of (a) single diffractive dijet production and (b) dijet production in double pomeron exchange in a $p \bar{p}$ interaction. 
The question which we seek to answer is whether hard diffraction processes obey QCD factorization, i.e. can be expressed in terms of the parton-parton cross section convoluted with a universal diffractive (anti)proton structure function. The diffractive structure function depends not only on $Q^{2}$ and the Bjorken scaling variable, $x$, but also on the fractional momentum loss of the (anti)proton, $\xi$, and the four momentum transfer squared at the $p-\mathbb{P}(\bar{p}-\mathbb{P})$ vertex, $t$. In this talk, factorization for hard diffraction processes is tested by comparing $p \bar{p}$ hard SD results with predictions from phenomenological models, expectations from results obtained in diffractive deep inelastic scattering (D-DIS) experiments, and results obtained in $p \bar{p}$ hard DPE studies.

\section{HARD DIFFRACTION WITH A RAPIDITY GAP}

The CDF collaboration has measured the fraction of events that contain forward rapidity gaps in $W$, dijet and $b$-quark production at $\sqrt{s}=1800 \mathrm{GeV}$ [5]. Recently, the $\mathrm{D} \varnothing$ collaboration has also reported a measurement of the gap fraction in forward and central dijet events at $\sqrt{s}=630$ and $1800 \mathrm{GeV}$ [1]. In the $\mathrm{D} \varnothing$ analysis, rapidity gaps are identified by using the $\mathrm{L} \phi$ forward scintillator arrays $(2.3<|\eta|<4.3)$, and a portion of the forward calorimeters $(3.0<|\eta|<5.2)$. The measured gap fractions are shown in Table 1 . The gap fractions predicted with four different pomeron structure functions, (1) "hard gluon", $s(\beta) \propto \beta(1-\beta)$; (2) "flat gluon", $s(\beta) \propto$ constant; (3) "soft gluon", $s(\beta) \propto(1-\beta)^{5}$; and (4) "quark", the quark analog of (1), are also shown in Table 1 , where $\beta(=x / \xi)$ is the momentum fraction of the Pomeron carried by the interacting parton. Although the quark structure is in general agreement with the data, it has previously been shown to yield a higher rate than that measured for diffractive $W$ production [5]. The lower half of Table 1 provides valuable information, since the Monte Carlo normalization cancels in the ratio of gap fractions for the same $\sqrt{s}$. A gluonic Pomeron containing significant soft and hard components, combined with a reduced (renormalized [6]) pomeron flux factor, could reasonably describe all the data samples.

TABLE 1. The measured and predicted gap fractions and their ratios.

\begin{tabular}{lccccc}
\hline \multicolumn{7}{c}{ Gap Fraction } \\
\hline Sample & Data & Hard Gluon & Flat Gluon & Soft Gluon & Quark \\
\hline $1800 \mathrm{GeV}|\eta|>1.6$ & $(0.65 \pm 0.04) \%$ & $(2.2 \pm 0.3) \%$ & $(2.2 \pm 0.3) \%$ & $(1.4 \pm 0.2) \%$ & $(0.79 \pm 0.12) \%$ \\
$1800 \mathrm{GeV}|\eta|<1.0$ & $(0.22 \pm 0.05) \%$ & $(2.5 \pm 0.4) \%$ & $(3.5 \pm 0.5) \%$ & $(0.05 \pm 0.01) \%$ & $(0.49 \pm 0.06) \%$ \\
$630 \mathrm{GeV}|\eta|>1.6$ & $(1.19 \pm 0.08) \%$ & $(3.9 \pm 0.9) \%$ & $(3.1 \pm 0.8) \%$ & $(1.9 \pm 0.4) \%$ & $(2.2 \pm 0.5) \%$ \\
$630 \mathrm{GeV}|\eta|<1.0$ & $(0.90 \pm 0.06) \%$ & $(5.2 \pm 0.7) \%$ & $(6.3 \pm 0.9) \%$ & $(0.14 \pm 0.04) \%$ & $(1.6 \pm 0.2) \%$ \\
\hline \multicolumn{6}{c}{ Ratio of Gap Fraction } \\
\hline $630 / 1800|\eta|>1.6$ & $1.8 \pm 0.2$ & $1.7 \pm 0.4$ & $1.4 \pm 0.3$ & $1.4 \pm 0.3$ & $2.7 \pm 0.6$ \\
$630 / 1800|\eta|<1.0$ & $4.1 \pm 0.9$ & $2.1 \pm 0.4$ & $1.8 \pm 0.3$ & $3.1 \pm 1.1$ & $3.2 \pm 0.5$ \\
$1800|\eta|>1.6 /|\eta|<1.0$ & $3.0 \pm 0.7$ & $0.88 \pm 0.18$ & $0.64 \pm 0.12$ & $30 . \pm 8$. & $1.6 \pm 0.3$ \\
$630|\eta|>1.6 /|\eta|<1.0$ & $1.3 \pm 0.1$ & $0.75 \pm 0.16$ & $0.48 \pm 0.12$ & $13 . \pm 4$. & $1.4 \pm 0.3$ \\
\hline
\end{tabular}




\section{DIFFRACTIVE DIJETS WITH A LEADING ANTIPROTON}

The CDF collaboration has studied SD dijet production at $\sqrt{s}=1800 \mathrm{GeV}[2]$ by using events triggered on a leading antiproton detected in a Roman Pot spectrometer (RPS). In this study, the diffractive structure function of the antiproton is measured and compared with expectations from results obtained in diffractive DIS experiments at HERA to test factorization. In leading order QCD, the ratio $R_{N D}^{S D}\left(x, Q^{2}, \xi\right)$ of diffractive to non-diffractive (ND) dijet rates is equal to the ratio of the diffractive to ND structure functions. The relevant diffractive structure functions integrated over $t$ can be written as $F_{j j}^{D}\left(x, Q^{2}, \xi\right)=$ $x\left[g^{D}\left(x, Q^{2}, \xi\right)+\frac{4}{9} q^{D}\left(x, Q^{2}, \xi\right)\right]$ where $g^{D}\left(x, Q^{2}, \xi\right)$ and $q^{D}\left(x, Q^{2}, \xi\right)$ are respectively the diffractive gluon and quark parton densities, and $\frac{4}{9}$ is a color factor. Therefore, the diffractive structure function can be obtained by multiplying the ratio $R_{N D}^{S D}\left(x, Q^{2}, \xi\right)$ by the known non-diffractive structure function $F_{j j}\left(x, Q^{2}\right)$. The value of $x$ is evaluated from the detected jets as $x=\sum_{i=1}^{2(3)} E_{T}^{i} e^{-\eta^{i}} / \sqrt{s}$, where the sum is carried out over the two leading (highest transverse energy) jets plus a third jet if $E_{T}^{j e t 3}>5 \mathrm{GeV}$. By changing variables, $x \rightarrow \beta \xi$, the obtained diffractive structure function $F_{j j}^{D}\left(x, Q^{2}, \xi\right)$ can be transformed to $F_{j j}^{D}\left(\beta, Q^{2}, \xi\right)$.

Figure 2 shows the measured $F_{j j}^{D}(\beta)$ for $0.035<\xi<0.095,|t|<1 \mathrm{GeV}^{2}$ and $E_{T}^{j e t 1,2}>7 \mathrm{GeV}$. The dashed (dotted) curve is the expectation for $F_{j j}^{D}(\beta)$ from fit 2 (fit 3) of the $\mathrm{H} 1$ diffractive parton densities [7] at $Q^{2}=75 \mathrm{GeV}^{2}$, which approximately corresponds to the $\left\langle E_{T}^{j e t}\right\rangle^{2}$ of the CDF data. The measured $F_{j j}^{D}(\beta)$ distribution and expectations from the $\mathrm{H} 1$ analysis of diffractive DIS events disagree both in normalization and shape, which indicates a breakdown of factorization. The discrepancy in normalization, defined as the ratio of the integral over $\beta$ of data to expectation, is $D=0.06 \pm 0.02(D=0.05 \pm 0.02)$ for fit 2 (fit 3 ).

\section{DIJET PRODUCTION IN DOUBLE POMERON EXCHANGE}

Dijet production in DPE has been studied by the CDF collaboration at $\sqrt{s}=$ $1800 \mathrm{GeV}$ [3] using events triggered on a leading antiproton and requiring a forward rapidity gap on the leading proton side.

As mentioned earlier, in leading order $\mathrm{QCD}$, the ratio $R_{N D}^{S D}\left(x_{\bar{p}}\right)$ of the SD to ND dijet events as a function of $x_{\bar{p}}$ is equal to the ratio of the diffractive to ND structure functions of the antiproton. Similarly, the ratio $R_{S D}^{D P E}\left(x_{p}\right)$ of the DPE to SD dijet rates as a function of $x_{p}$ is equal to the ratio of the diffractive to ND structure functions of the proton. The variables $x_{p}$ and $x_{\bar{p}}$ are the Bjorken scaling variables for the proton and antiproton, respectively. Therefore, factorization for hard diffraction processes can be tested by comparing $R_{N D}^{S D}\left(x_{\bar{p}}\right)$ with $R_{S D}^{D P E}\left(x_{p}\right)$. A 


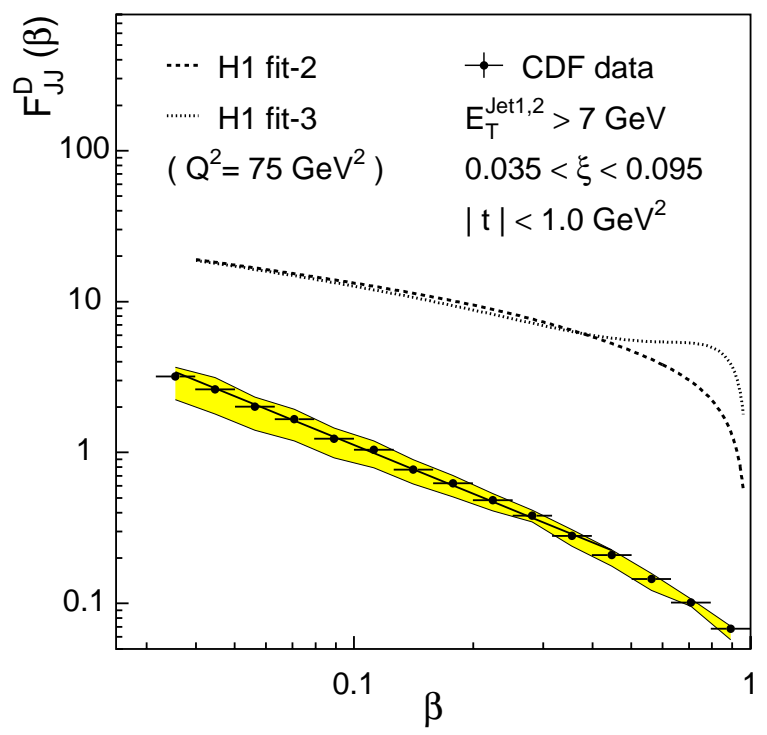

FIGURE 2. Data $\beta$ distribution (points) compared with expectations from the parton densities of the proton extracted from diffractive deep inelastic scattering by the $\mathrm{H} 1$ collaboration. The straight line is a fit to the data of the form $\beta^{-n}$ in the region $\beta<0.5$. The lower (upper) boundary of the filled band represents the data distribution obtained by using only the two leading jets (up to four jets of $E_{T}>5 \mathrm{GeV}$ ) in evaluating $\beta$. The dashed (dotted) lines are expectations from the H1 fit 2 (fit 3 ). The systematic uncertainty in the data normalization is $\pm 25 \%$.

deviation of the double ratio $D=R_{N D}^{S D}\left(x_{\bar{p}}\right) / R_{S D}^{D P E}\left(x_{p}\right)$ from unity would indicate a breakdown of factorization.

In figure 3, the ratio $R_{S D}^{D P E}\left(x_{p}\right)$ is compared with $R_{N D}^{S D}\left(x_{\bar{p}}\right)$ as a function of $x(\equiv$ $\left.x_{p}=x_{\bar{p}}\right)$. The data are restricted to the regions $7<E_{T}^{\text {jet1,2 }}<10 \mathrm{GeV},\left|t_{\bar{p}}\right|<$ $1 \mathrm{GeV}^{2}, 0.035<\xi_{\bar{p}}<0.095$, and for DPE $0.01<\xi_{p}<0.03$, where $\xi_{\bar{p}}\left(\xi_{p}\right)$ is the fractional momentum loss of the antiproton (proton), and $t_{\bar{p}}$ is the four momentum transfer squared at the $\bar{p}-\mathbb{P}$ vertex. Both ratios are normalized per unit $\xi$. Although the factorization test has to be performed at the same $x$ and $\xi$ values, the $\xi_{\bar{p}}$ and $\xi_{p}$ regions in the data do not overlap. The $\xi_{\bar{p}}$ dependence of the ratio $\tilde{R}_{N D}^{S D}$ is examined in the insert of figure 3 , where the tilde over the $R$ indicates the weighted average in the region of $x$ within the vertical dashed lines in the main figure. The ratio $\widetilde{R}_{N D}^{S D}$ is found to be flat in the region $0.035<\xi_{\bar{p}}<0.095$. The ratio of $\tilde{R}_{N D}^{S D}$ extrapolated down to $\xi_{\bar{p}}=0.02$ to be compared with $\tilde{R}_{S D}^{D P E}$ in the region $0.01<\xi_{p}<0.03$ is $D \equiv \tilde{R}_{N D}^{S D} / \tilde{R}_{S D}^{D P E}=0.19 \pm 0.07$, which indicates a breakdown of factorization.

\section{CONCLUSION}

The CDF and D $\varnothing$ collaborations have studied hard single diffraction and double pomeron exchange events produced in $p \bar{p}$ collisions at the Fermilab Tevatron 


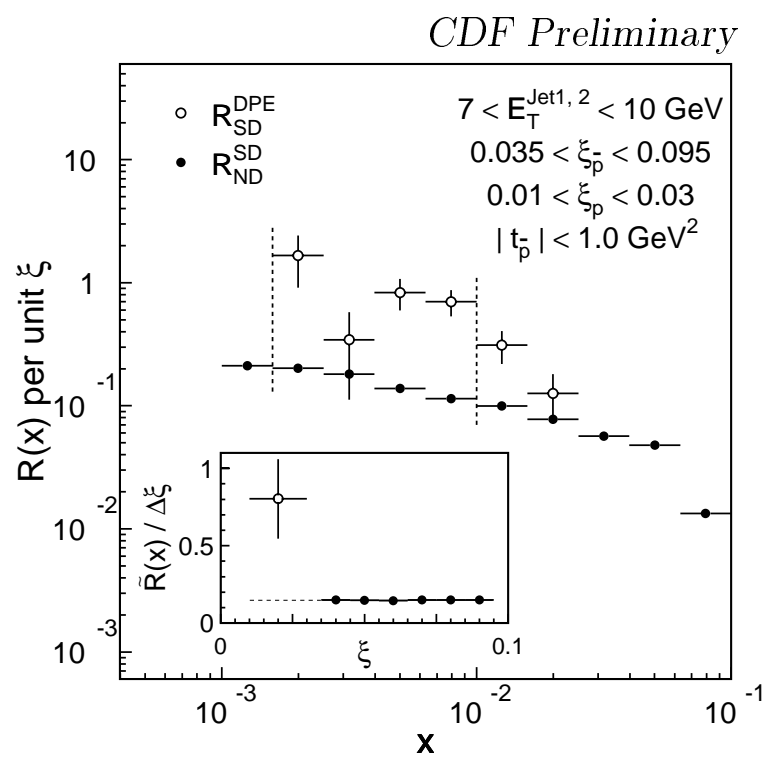

FIGURE 3. Ratios of DPE to SD (SD to ND) dijet event rates per unit $\xi$, shown as open (filled) circles, as a function $x$-Bjorken of partons in the $p(\bar{p})$. The errors are statistical only. The SD/ND ratio has a normalization uncertainty of $\pm 20 \%$. The insert shows $\tilde{R}(x)$ per unit $\xi$ versus $\xi$, where the tilde over the $R$ indicates the weighted average of the $R(x)$ points in the region of $x$ within the vertical dashed lines, which mark the DPE kinematic boundary (left) and the value of $x=\xi_{p}^{\text {min }}$ (right).

collider. A breakdown of factorization for hard diffraction processes is observed in comparing $p \bar{p}$ hard single diffraction results with expectations from results obtained in diffractive deep inelastic scattering experiments, or with results from a study of $p \bar{p}$ hard double pomeron exchange events. These studies provide further insight into the mechanism of hard diffraction, which will undoubtedly help in establishing the phenomenology of hadronic diffraction processes.

\section{REFERENCES}

1. B. Abbott et al., (D $\varnothing$ Collaboration), FERMILAB-PUB-99/375-E.

2. T. Affolder et al., (CDF Collaboration), Phys. Rev. Lett. 84, 5043 (2000).

3. T. Affolder et al., (CDF Collaboration), FERMILAB-PUB-00/098-E.

4. The pseudorapidity, $\eta$, is defined as $\eta=-\ln \left(\tan \frac{\theta}{2}\right)$, where $\theta$ is the polar angle between a particle and the proton beam direction.

5. F. Abe et al., (CDF Collaboration), Phys. Rev. Lett. 78, 2698 (1997); 79, 2636 (1997);

T. Affolder et al., Phys. Rev. Lett. 84, 232 (2000).

6. K. Goulianos, Phys. Lett. B 358, 379 (1995); B 363, 268 (1995).

7. C. Adloff et al., (H1 Collaboration), Z. Phys. C 76, 613 (1997) 